\title{
Differences in virus prevalence and load in the hearts of patients with chronic dilated cardiomyopathy with and without immune- mediated inflammatory disease
}

\author{
R Dennert ${ }^{1 *}, \mathrm{P}$ van Paassen ${ }^{2}, \mathrm{C}$ Bruggeman ${ }^{3}, \mathrm{P}$ Wolffs $^{3}, \mathrm{~J}$ W Cohen Tervaert ${ }^{2}$, S Heymans ${ }^{1}$ \\ From 5th European Workshop on Immune-Mediated Inflammatory Diseases \\ Sitges-Barcelona, Spain. 1-3 December 2010
}

\section{Introduction}

Autoimmune responses against the heart and infections with cardiotropic viruses have been suggested to play a major role in the pathogeneses of idiopathic dilated cardiomyopathy (DCM). The interaction and cross-talk between these complex mechanisms is not completely understood, making etiologic distinction difficult.

\section{Aim}

We compared the prevalence and quantity of cardiotropic viruses in heart tissue of DCM patients with and without a previously diagnosed immune mediated inflammatory disorder (IMID).

\section{Patients and methods}

Myocardial tissue samples and serum was obtained from 159 consecutive patients with DCM and 20 controls. Patients were subdivided into groups based on the absence $(n=125)$ or presence $(n=34)$ of an IMID, and controls $(n=20)$.

\section{Results}

The IMID patients showed elevated serum soluble interleukin-2 receptor and neopterin compared to the nonautoimmunity patients and controls, compatible with the fact that these patients had an increased cellular immune activation related to their IMID.

The non-IMID group revealed a higher PVB19 prevalence $(100 / 125)$ compared to the autoimmunity patients $(16 / 34, \mathrm{p}=0.04)$ and controls $(11 / 20, \mathrm{p}=0.02)$ and PVB19 copy numbers (561 \pm 97 vs. $191 \pm 92$ copies/ $\mu$ g DNA, and $103 \pm 47$ copies/ $\mu$ g DNA, respectively, both $\mathrm{p}<0.001$ ).

Both the non-IMID and IMID DCM patients demonstrated increased myocardial inflammation compared to controls $(12.5 \pm 1.8$ and $14.0 \pm 3.2$ vs. $5.1 \pm 0.7$ CD $45-$ positive inflammatory cells, both $\mathrm{p}<0.05)$.

\section{Conclusion}

Our data shows a similar PVB19 prevalence and load in hearts of autoimmunity DCM patients and controls, but increased prevalence and levels in non-autoimmunity DCM patients. These findings suggest that ICM patients in the presence of an IMID have a different pathophysiologic mechanism compared to the virus-induced form of ICM.

\section{Author details}

'Dept. of Cardiology, Cardiovascular Research Institute Maastricht, Maastricht University Medical Center, Maastricht, The Netherlands. ${ }^{2}$ Division of Clinical and Experimental Immunology, Dept. of Internal Medicine, Maastricht University Medical Center, Maastricht, The Netherlands. ${ }^{3}$ Dept. of Medical Microbiology, Maastricht University Medical Center, The Netherlands.

Published: 25 November 2010

doi:10.1186/1479-5876-8-S1-P11

Cite this article as: Dennert et al:: Differences in virus prevalence and load in the hearts of patients with chronic dilated cardiomyopathy with and without immune-mediated inflammatory disease. Journal of Translational Medicine 2010 8(Suppl 1):P11. 